\title{
Helminth infection in southern Laos: high prevalence and low awareness
}

Khampheng Phongluxa ${ }^{1,2,3}$, Vilavanh Xayaseng ${ }^{1,2,3}$, Youthanavanh Vonghachack ${ }^{2,3,5}$, Kongsap Akkhavong ${ }^{1}$, Peter van Eeuwijk $k^{2,3,4}$ and Peter Odermatt ${ }^{2,3^{*}}$

\begin{abstract}
Background: Helminthiasis is a public health concern in Lao People's Democratic Republic (Lao PDR, Laos). This study aimed to understand helminth infection and associated risk factors in relation to existing local knowledge, attitudes and practices regarding worm infections in endemic communities.
\end{abstract}

Methods: A cross-sectional survey was conducted in 10 randomly selected villages in Saravane district, Southern Laos. Two stool samples obtained from 574 members (aged $\geq 2$ years) of selected households were examined using the Kato Katz method. Household heads $(n=130)$ were interviewed. Eight focus group discussions (FGDs) and direct observations were performed. Uni- and multivariate logistic regression analyses were conducted to predict infection. Content analysis was conducted for qualitative data.

Results: The prevalence of Opisthorchis viverrini, hookworm, Trichuris trichiura, Ascaris lumbricoides and Taenia sp. was $88.7 \%, 86.6 \%, 32.9 \%, 9.8 \%$ and $11.5 \%$, respectively. Most individuals were co-infected with O. viverrini and hookworm. More men had multiple helminth infections than did women. Only one-third of household heads had heard about liver fluke before, of which $59.2 \%$ associated it with eating raw fish dish. Among the soil-transmitted helminths, roundworm was the most well known (70.8\%) but was attributed to raw food consumption (91.3\%). Eating raw fish was a common practice (75.4\%); few households possessed a latrine (16.1\%); less than half of the study participants mentioned health benefits from latrine use and personal hygiene. Focus group discussion participants had a low level of awareness of worm infections; more men liked eating raw fish than did women; some disliked using latrines because they were not used to it and because of their bad smell. Poor personal and village hygiene practices were observed.

Conclusions: This study highlights a high helminth infection rate and poor community awareness of worm infections and associated risk factors. Only a sound awareness of worm infection and the underlying risk factors may prevent infection and re-infection after treatment.

Keywords: Helminth infection, Soil-transmitted helminth, Liver fluke, Raw fish consumption, Latrine use, Health education

\section{Background}

Food-borne trematodiases (FBT) are among the neglected tropical diseases [1] and a public health problem in many parts of the world, with a global burden of 665,000 disability adjusted life years [2]. FBT often coexists with soil-transmitted helminthiasis (STH) [3,4]. About 750 million people are at risk for FBT, of which

\footnotetext{
*Correspondence: peter.odermatt@unibas.ch

${ }^{2}$ Department of Epidemiology and Public Health, Swiss Tropical and Public Health Institute, P.O. Box, CH-4002, Basel, Switzerland

${ }^{3}$ University of Basel, Basel, Switzerland

Full list of author information is available at the end of the article
}

40 million are infected [5]. An estimated 1.2 billion, 800 million and 740 million people worldwide are infected with Ascaris lumbricoides, Trichuris trichiura, and hookworm, respectively [6]. In Southeast Asia, about 67.3 million people are at risk for Opisthorchis viverrini, the most frequently observed FBT. Ten million people are infected in Thailand and Laos [7]. O. viverrini infection is common where consumption of raw or insufficiently cooked fish dishes is deeply rooted in local culture and where proper sanitation is minimal or absent [1]. O. viverrini infection results in hepatobiliary morbidity 
[2] and chronic infection may lead to cholangiocarcinoma, a fatal bile duct cancer [8]. Preventive chemotherapy together with health education is the most current helminth control strategy [9-11]. However, re-infection rates after treatment with anti-helminthic drugs are rapidly reaching pre-treatment prevalence rates [12] as food and hygiene behaviour patterns remain unchanged, indicating that health education is not effectively addressing the critical issues.

Deepening our understanding of helminth infections in relation to local notions of these infections, its risk factors and deworming measures is important for developing sustainable and effective helminth control. For example, in northern Vietnam, local communities were aware of the risks of eating raw fish dish, however, people were less cautious about consuming raw fish dishes because, they argued, with the effective drug, they could be easily cured after an infection [13]. Having a good knowledge of worm infection and of people's perception of the risk factors and of the benefits of deworming facilitates the development of valid interventions and convinces people to comply with the intervention [14-17]. Hence, an in-depth knowledge of infection prevalence and of the risks of endemic helminths and an understanding of corresponding local knowledge and perception patterns is essential for improving helminth control activities in the local setting.

In Laos, over two million people are infected with liver fluke [7]. Recent surveys reveal a rampant $O$. viverrini infection rate of more than $90 \%$ of the general population in southern areas [18-20]. About 1.6 million children under 15 years of age are at risk of STHs. However, our understanding of the local knowledge, attitudes and practices in relation to worm infection and its risk factors is limited. Today, preventive chemotherapy combined with health education via vertical programmes remains the main strategy for helminth control in Laos. It is implemented without detailed knowledge of the attitudes and practices of the local communities. Therefore, underlying risk factors such as food preparation and hygiene behaviour remain inadequately addressed and, hence, re-infection rates are high.

This study aimed to advance our understanding of helminth infection in relation to the community's existing knowledge, attitudes and practices in endemic areas in southern Laos. A cross-sectional survey was conducted in randomly selected villages and households in Saravane district to assess helminth infections and their risk factors. Focus group discussions (FGDs) and direct observations were carried out to triangulate knowledge, attitudes and practices with regard to helminth infections.

\section{Methods}

\section{Ethics statement}

Ethical clearance was obtained from the National Ethics Committee on Health Research, Ministry of Health, Lao PDR (Ethical Clearance No 169/NECHR, 1 April 2008). Written informed consent was obtained from heads of selected households. For illiterate persons, informed consent was read out and, after approval, the person signed with a fingerprint. The interviewees were informed about the study aim, the study procedures, the need for voluntarily participation, and their right to stop participation at any time. All infections diagnosed in this study were treated according to the national treatment guidelines [21].

\section{Study areas and population}

The study was conducted in ten randomly selected villages of Saravane district, Saravane province (Figure 1). The total population in these villages comprised 6,207 inhabitants (including 3,056 females and 847 children under five years of age). Some villages were located near rivers, namely Xedonh and Xeseth, and their tributaries (Houay Lanong, Houay Namxai, and Houay Nongboua). In some villages, the Laotheung ethnic group formed the majority of the population. The main occupation of residents was subsistence rice farming. Daily food intake consisted of rice with vegetables and bamboo, as well as fish from rivers and ponds and game. In four villages (Nakhoisao, Nahinlong, Naphengyai, and Hangphounoy), demonstration latrines were constructed by the Saravane Provincial Health Office and international projects.

Saravane district is located on the Bolaven Plateau. It covers $2,441 \mathrm{~km}^{2}$ and has a population density of three people per square kilometre $\left(\mathrm{km}^{2}\right)$. The 89,068 inhabitants (female: 45,520 ) live in 178 villages and in 13,239 households. Ethnic groups include the Laotheung and the Laoloum. The annual income per capita is USD 627. There are nine health centres, which cover $71 \%$ of the villages. The district has 19 pharmacies, 91 villages with drug kits, two private clinics and 13 outreach teams (Report Saravane District Health Office [DHO], 2008). In 2002, a national parasitology survey indicated that $21.5 \%, 11.9 \%, 10.9 \%$ and $5.4 \%$ of primary school children in Saravane province were infected with O. viverrini, $A$. lumbricoides, hookworm and T. trichiura, respectively [22]. In 2004, the prevalence of O. viverrini infection was estimated at $58.5 \%$ (village range from $20.0 \%$ to $85.5 \%)$ in Saravane district. The infection rate increased with age. Eating raw or insufficiently cooked fish dishes was very common (79.7\%). Only one in 13 villages had latrines. Sixty per cent of the cyprinoid fish species consumed in this district are infected with $O$. viverrini metacercariae [23]. Mass treatment against helminth 


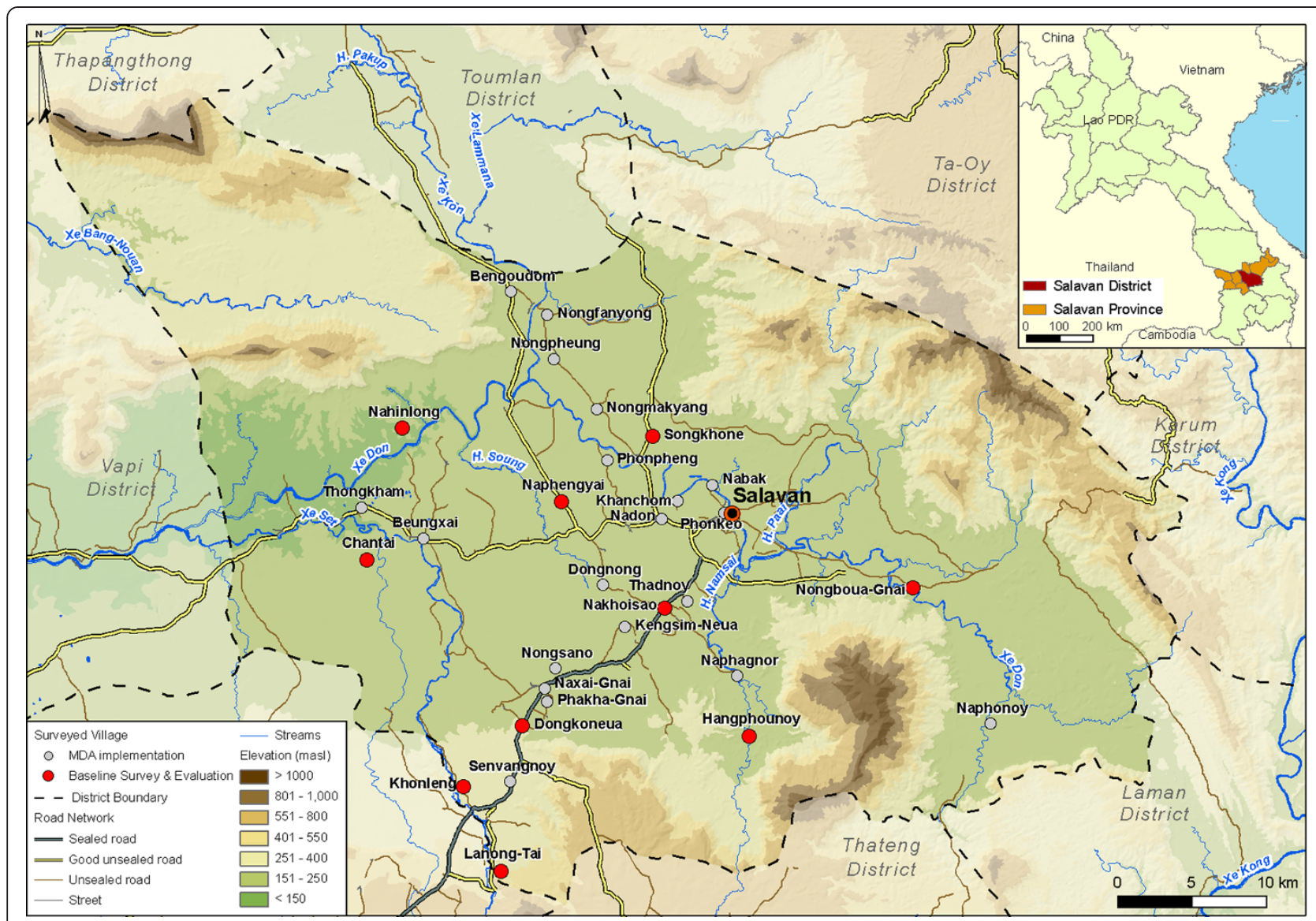

Figure 1 Study villages (red spots) in Saravane district, province of Saravane, Lao PDR.

infection does not yet exist in Saravane district (Director of Saravane Provincial Health Office [PHO]).

\section{Study design}

A cross-sectional survey was carried out in January and February 2010, using two-stage cluster sampling. First, 10 villages were randomly selected from the list of villages in the district obtained from the Saravane DHO. Second, 20 households were randomly selected from the household list. All household members aged 2 years and older were enrolled and intestinal helminth infections were diagnosed from two stools samples. Heads of households were interviewed. Eight FGDs were carried out in four randomly selected villages; in each village two FGDs were conducted: one among men and another among women. Direct observations were performed in all villages.

\section{Field and laboratory procedures}

Village leaders and household heads were informed about the study's objectives and procedures. A structured interview was conducted with heads of selected households, where information on socio-demographic household characteristics, household assets, and knowledge, attitudes and practices relating to $O$. viverrini and helminth infections (e.g. raw fish consumption, hygiene and sanitation behaviour) were collected. FGD participants were randomly selected from households that were not part of the cross-sectional survey. In FGDs, openended and open questions were used, i.e. (i) Have you heard about liver fluke/roundworm/hookworm/whipworm? (ii) If yes, could you please tell us how people get this infection? (iii) What is the relationship between infection and disease? (iv) How can you prevent it? (v) Do you eat raw fish dishes? How often? (vi) What do you think about eating raw fish dishes? (vii) Do you have a latrine? What do you think about using latrines? (viii) What are the benefits of using a latrine and of hand washing before eating/after defecation? (ix) What do you think about hand washing before eating and after defecation? (x) Do you wash your hands before eating and/or after defecation? Team leaders made direct observations during visits in the communities regarding the cleanliness of the village, the consumption of raw fish dish, personal hygiene practices, wearing shoes and the presence of sanitation facilities. 
In the cross-sectional study, for each enrolled household member, two stool samples were collected on two consecutive days and examined. Stool containers labelled with date, ID, name, age and sex of participant were handed-out. Heads of households were instructed on how to fill the container with stool samples. On the collection day, people who gave a first stool sample received a second pre-labelled container to use for the following day. All collected samples were kept in a cool box and were transported by car to the provincial hospital's laboratory in Saravane within an hour after collection. For each stool sample, one Kato Katz thick smear slide was created, using standard $41.7 \mathrm{mg}$ templates [24]. After a clearance time of 30 minutes, the slide was examined under a light microscope $(100 \times$ magnification $)$. All samples were examined on the day of collection. The number of eggs per parasite was counted and recorded for each parasite species separately.

\section{Data management and statistical analysis}

Data were entered twice (double entry) into EpiData, version 3.1 (Epidata Association; Odense, Denmark) and validated. Analysis was performed using Stata software, version 10.1 (Stata Corp., College Station, TX, USA). Only participants with two stool samples were retained in the analysis. Individuals were divided into seven age groups (in year) $(<6,6-15,16-29,30-39,40-49,50-59$ and 60+); most participants were aged between 6-15 (26.8\%), 16-29 (21.8\%) and 30-39 (16.0\%). Descriptive statistics were calculated (counts, percentages, means and standard deviations $[\mathrm{SD}]$ ). The intensity of helminth infections was expressed in terms of egg count per gram faeces (EPG). According to Maleewong et al. and Sayasone et al. [23,25], for the infection with $O$. viverrini, and STHs the following light, moderate, and high intensity groups were established based on the EPG counts: Hookworm, 1-1999 EPG, 2000-3999 EPG, and $\geq 4000$ EPG; A. lumbricoides, 1-4999 EPG, 500049,999 EPG, and $\geq 50,000 \mathrm{EPG}$; and T. trichiura and O. viverrini, 1-999 EPG, 1000-9999 EPG, and $\geq 10,000$ EPG. Pearson's $x^{2}$ and Fisher's exact tests were used to compare proportions. The association between O. viverrini infection and sex, age group, ethnicity, raw fish consumption, having a latrine, and socio-economic status (SES) was assessed in a univariate logistic regression analysis. Predictors with $\mathrm{p}<0.25$ were retained in a multivariate logistic regression model. Odds ratio (OR) and 95\% confidence intervals (95\% CI) were reported. P-values less than $5 \%$ were considered significant.

SES of households was assessed using Multiple Correspondence Analysis (MCA). The technique has been used before for categorical data [18]. Household assets, materials used for house construction, water source and possession of a latrine were used to construct a socio- economic index. Households were classified into one of five quintiles: most poor, very poor, poor, less poor and least poor, using the socio-economic index.

Qualitative data from FGDs were transcribed from notes and tape recordings, then translated from Lao into English, typed into MSWord and imported to MAXQDA (version 10) software for textual analysis. Statements were coded and categorised according to the following themes: knowledge of liver fluke, hookworm, whipworm, roundworm and tapeworm; raw fish consumption; hand washing before eating; hand washing after latrine use and deworming. Coded data were retrieved and exported to MSExcel for frequency and content analysis.

\section{Results}

\section{Characteristics of the study population}

One hundred and thirty heads of households were retained in the analysis (Figure 2); $53.1 \%$ were male; the mean age was 41.2 years (SD 13.6 years, range: 20-81 years); mean age by gender was 43.4 years (SD 14.6 years; range $20-81$ years) for men and 38.5 years (SD 11.8 years; range 21-74 years) for women. Almost all household heads were farmers (93.1\%); $56.9 \%$ had completed primary school while $29.2 \%$ had not attended school; $56.9 \%$ belonged to the Laoloum ethnic group.

From the enrolled households, 574 people submitted two stool samples (Figure 2); $50.3 \%$ of these were from females; the mean age was 25.5 years (SD 18.2 years, range: $2-81$ years). There was no significant age difference between genders: the mean age for males was 24.4 years (SD 18.2 years, range $2-81$ years, $95 \%$ CI 22.3-26.5) and 26.6 years (SD 18.3 years, range 2-74 years, 95\% CI 24.5-28.7) for females; $53.3 \%$ belonged to the Laoloum ethnic group.

Among 67 FGD participants, 52.2\% were females. The mean age was 40.6 years (range 15-73 years). Most of them were in the age groups of 35-44 years (32.8\%) and 25-34 years (22.4\%); more than two-third of the FGD participants belonged to the Laotheung ethnic group (71.6\%) and were Buddhists (71.7\%); 55.2\% had attended primary school while $40.3 \%$ had not attended school at all. All were farmers.

\section{Helminth infection prevalence, intensity and risk factors}

Intestinal helminth infections were highly prevalent. O. viverrini, hookworm, T. trichiura and A. lumbricoides were diagnosed in $88.7 \%, 86.6 \%, 32.9 \%$ and $9.8 \%$ of the individuals, respectively. In addition, Taenia sp. was detected in $11.5 \%$ of the study participants. O. viverrini infection intensity increased with age and reached a mean infection intensity of 2163 EPG in the oldest age group. $O$. viverrini, hookworm and $A$. lumbricoides infection rates were not significantly different between genders 


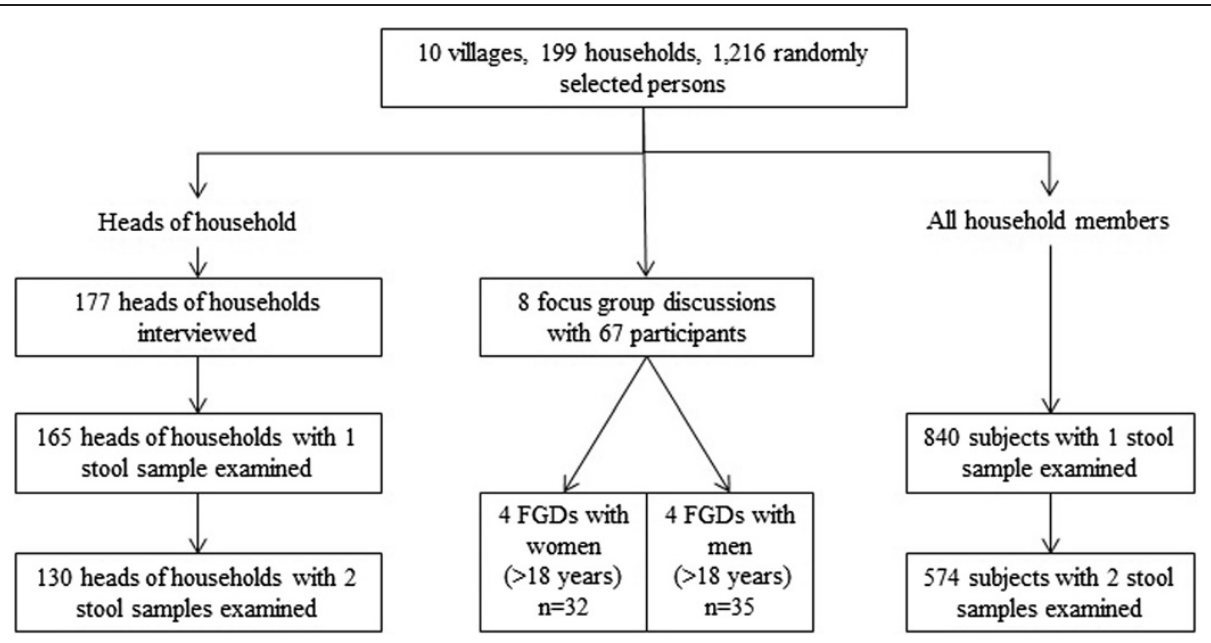

Figure 2 Study diagram, Saravane district, 2010.

but $T$. trichiura infection was significantly more frequent in male participants than in female participants $(\mathrm{p}=0.031$, Table 1$)$.

Prevalence of $O$. viverrini and hookworm increased significantly with age, from $72.6 \%$ to $100 \%(\mathrm{p}<0.001)$ and from $71.2 \%$ to $96.7 \%(\mathrm{p}=0.001)$ from the youngest to the oldest age group, respectively, whereas Taenia sp. increased from $6.8 \%$ at ages $<6$ years to $21.4 \%$ among people aged $50-59$ and then declined to $13.3 \%$ at ages $>59$ $(\mathrm{p}=0.034$, Figure 3$)$.

Multiparasitism was very common. More than half of the participants (52.4\%) harboured two helminth species or more. Three different species were diagnosed in $27.2 \%$ of the participants. Infection with three helminth species was detected in males significantly more (33.3\%) than in females $(21.1 \%, \mathrm{p}=0.005$, Figure 4$)$. Among $O$. viverrini-infected people, more than three quarters (77.3\%) were co-infected with hookworm, of which $74.7 \%$ were children in the age group 6-15 years.

\section{Risk factors of $O$. viverrini infection}

After an initial univariate prediction of O. viverrini, hookworm, T. trichiura and A. lumbricoides infection in all study participants, the following variables were included in the multivariable logistic regression analysis model for predicting hookworm: gender, age group, ethnicity and having a latrine; only the first three variables were included for predicting O. viverrini and A. lumbricoides and only gender and ethnicity were included for predicting T. trichiura. Results indicated that males had a strongly increased risk for $O$. viverrini infection compared to females $(\mathrm{OR}=1.9, \mathrm{p}=0.023)$. Laoloum had a significantly higher risk for $O$. viverrini infection than did Laotheung $(\mathrm{OR}=2.3, \mathrm{p}=0.003)$ but a lower risk for hookworm infection $(\mathrm{OR}=0.59, \mathrm{p}=0.049)$, $T$. trichiura
$(\mathrm{OR}=0.25, \mathrm{p}<0.001)$ and $A$. lumbricoides $(\mathrm{OR}=0.27$, $\mathrm{p}<0.001)$ compared to Laotheung. Children aged $<6$ years were at significantly lower risk for $O$. viverrini $(\mathrm{OR}=0.12$, $\mathrm{p}=0.006)$ and hookworm infection $(\mathrm{OR}=0.08, \mathrm{p}=0.015)$ compared to older age groups. In contrast, they had a significantly higher risk of being infected with $A$. lumbricoides than did other age groups $(\mathrm{OR}=5.31, \mathrm{p}=0.035$; Table 2).

With regards to knowledge of helminth infection and its risk factors, the following variables were retained as predictors for an $O$. viverrini infection in the multivariable logistic regression model: having heard about O. viverrini, eating raw fish salad, eating raw fermented fish and eating sour rice-fermented fish. Results showed that only eating raw fish salad was associated with the $O$. viverrini infection: those who reported eating raw fish salad (Koi Pa, Lap Pa Nuew) had a highly increased risk for infection compared to those who did not eat raw fish salad $(\mathrm{OR}=16.1, \mathrm{p}=0.026)$.

\section{Knowledge, attitudes and practices of heads of households}

Heads of households were interviewed about their knowledge of worm infection and prevention. Questions about liver fluke, hookworm, whipworm and roundworm were asked. Of the interviewees, $37.7 \%$ had heard about liver fluke before, named "pha yat bai mai nai tap" or "san tap" in Lao. A little more than half (59.2\%) linked the infection to eating raw or insufficiently cooked fish dish, but only about a quarter of them linked it to the consumption of raw fermented fish sauce (24.5\%) and raw sour rice-fermented fish (24.5\%). Few household heads knew how to prevent liver fluke infections (14.3\%). Among the STHs, roundworm was most wellknown (70.8\%). However, almost all of those people who knew of it (91.3\%) attributed it to consumption of any 
Table 1 Helminth infection prevalence and intensity by gender and age group (in years), Saravane district, 2010 ( $n=574$ )

\begin{tabular}{|c|c|c|c|c|c|c|c|c|c|c|}
\hline Item & n (\%) & Male & Female & $<6$ & $6-15$ & $16-29$ & $30-39$ & $40-49$ & $50-59$ & $60+$ \\
\hline Opisthorchis viverrini & $509(88.7)$ & 91.2 & 86.1 & 72.6 & 87.0 & 88.8 & 91.3 & 98.3 & 95.2 & 100 \\
\hline GM faecal egg count (range), EPG: & $925^{*}(24-69,648)$ & 1100.3 & 771.5 & 221.6 & 406.0 & 1337.9 & 1388.7 & 2508.7 & 1890.7 & 2162.9 \\
\hline \multicolumn{11}{|l|}{ Intensity infection: } \\
\hline Light (1-999 EPG) & $261(51.3)$ & 47.7 & 55.0 & 88.7 & 70.9 & 45.0 & 36.9 & 28.1 & 30.0 & 33.3 \\
\hline Moderate (1000-9999 EPG) & $184(36.1)$ & 38.1 & 34.1 & 9.43 & 23.9 & 40.5 & 48.8 & 49.1 & 50.0 & 43.3 \\
\hline Heavy ( $\geq 10000$ EPG) & $64(12.6)$ & 14.2 & 10.8 & 1.9 & 5.2 & 14.4 & 14.3 & 22.8 & 20.0 & 23.3 \\
\hline Hookworm & $497(86.6)$ & 88.8 & 84.4 & 71.2 & 87.0 & 86.4 & 92.4 & 86.2 & 92.9 & 96.7 \\
\hline GM faecal egg count (range), EPG: & $446.5^{*}(24-38,880)$ & 492.2 & 403.6 & 342.1 & 393.5 & 443.9 & 538.7 & 381.4 & 543.1 & 768.5 \\
\hline \multicolumn{11}{|l|}{ Intensity of infection: } \\
\hline Light (1-1999 EPG) & $426(85.7)$ & 84.6 & 86.9 & 90.4 & 86.6 & 88.9 & 77.6 & 96 & 79.5 & 75.9 \\
\hline Moderate (2000-3999 EPG) & $33(6.6)$ & 8.3 & 4.9 & 3.8 & 7.5 & 3.7 & 10.6 & 0.0 & 15.4 & 6.9 \\
\hline Heavy ( $\geq 4000$ EPG) & $38(7.7)$ & 7.1 & 8.2 & 5.8 & 5.9 & 7.4 & 11.8 & 4.0 & 5.1 & 17.2 \\
\hline Trichuris trichiura & $189(32.9)$ & 37.5 & 28.4 & 38.4 & 35.1 & 29.6 & 29.4 & 37.9 & 33.3 & 23.3 \\
\hline GM faecal egg count (range), EPG: & $189.6^{*}(24-10,800)$ & 179.9 & 202.9 & 187.9 & 179.1 & 221.4 & 156.3 & 253.5 & 128.8 & 246.2 \\
\hline \multicolumn{11}{|l|}{ Intensity of infection: } \\
\hline Light (1-999 EPG) & $164(86.8)$ & 85.9 & 87.8 & 92.9 & 81.5 & 86.5 & 88.9 & 90.9 & 92.9 & 71.4 \\
\hline Moderate (1000-9999 EPG) & $24(12.7)$ & 14.0 & 10.9 & 7.1 & 16.7 & 13.5 & 11.1 & 9.1 & 7.1 & 28.6 \\
\hline Heavy ( $\geq 10000$ EPG) & $1(0.5)$ & 0 & 1.2 & 0 & 1.8 & 0 & 0 & 0 & 0 & 0 \\
\hline Ascaris lumbricoides & $56(9.8)$ & 8.1 & 11.4 & 19.2 & 8.4 & 9.6 & 7.6 & 8.6 & 4.8 & 10.0 \\
\hline GM faecal egg count (range), EPG: & $2984.5^{*}(96-178,080)$ & 3031.2 & 2952.4 & 6566.2 & 6691.1 & 757.1 & 4416.9 & 656.1 & 1515.4 & 4321.5 \\
\hline \multicolumn{11}{|l|}{ Intensity of infection: } \\
\hline Light (1-4999 EPG) & $35(62.7)$ & 65.2 & 60.6 & 42.9 & 53.8 & 83.3 & 57.1 & 100 & 50.0 & 66.7 \\
\hline Moderate (5000-49999 EPG) & $15(26.8)$ & 21.7 & 30.3 & 42.9 & 23.1 & 16.7 & 28.6 & 0 & 50.0 & 33.3 \\
\hline Heavy ( $\geq 50000$ EPG) & $6(10.7)$ & 13.0 & 9.1 & 14.3 & 23.1 & 0 & 14.3 & 0 & 0 & 0 \\
\hline Taenia sp. & $66(11.5)$ & 11.6 & 11.4 & 6.8 & 5.8 & 13.6 & 14.1 & 15.5 & 21.4 & 13.3 \\
\hline
\end{tabular}

$\mathrm{GM}=$ geometric mean; $\mathrm{EPG}=$ egg per gram of stool; * = mean of parasite egg.

type of raw food. Only a few people mentioned correct preventive measures (Table 3).

Among household heads, $43.1 \%$ thought that hand washing before eating could prevent diseases such as abdominal pains and diarrhoea. Others (21.5\%) did not see any link to health. A few household heads (2.3\%) reported that in order to prevent worm infections, people should wash their hands before eating.

Only a few household heads (16.1\%) reported having a latrine. Among them, females (92.3\%) used them significantly more frequently than did male household heads $(50.0 \%, \mathrm{p}=0.047$; Table 4$)$. About a quarter $(26.1 \%)$ of household heads thought that the possession of a latrine would be convenient, as one would not get wet from rain and would not need to go to the forest. About a quarter (24.6\%) of them believed that latrine possession could prevent disease transmission. Some heads of households (15.4\%) understood that latrine use could prevent diarrhoea or even malaria.
Raw food consumption was very common (Table 4). Among household heads, $75.4 \%$ reported regularly consuming raw fish dishes (Koi Pa, Lap Pa Nuew), 68.1\% ate sour rice-fermented fish (Som Pa, Som Pa Noy/Som $\mathrm{Pa}$ Chom) and $45.4 \%$ ate fermented fish sauce ( $\mathrm{Pa}$ Deak, $\mathrm{Pa}$ Deak Nam). Many more men reported eating raw fish dish and other raw food dishes than did females (91.3\% versus 57.4\%; $\mathrm{p}<0.001)$.

\section{Focus group discussion results}

Among the 67 FGD participants, 50.7\% reported having heard about liver fluke infection before; $43.3 \%$ associated the infection with eating any type of raw food and $4.5 \%$ linked the infection to eating raw vegetables; $11.9 \%$ related the consumption of raw or insufficiently cooked fish to a worm infection. Roundworm was the most well-known STH (77.6\%; Table 5).

Half of the participants (49.3\%) who reported repeatedly eating raw fish dishes explained this issue. A man (aged 31 years) in Naphengyai village said that he loved 


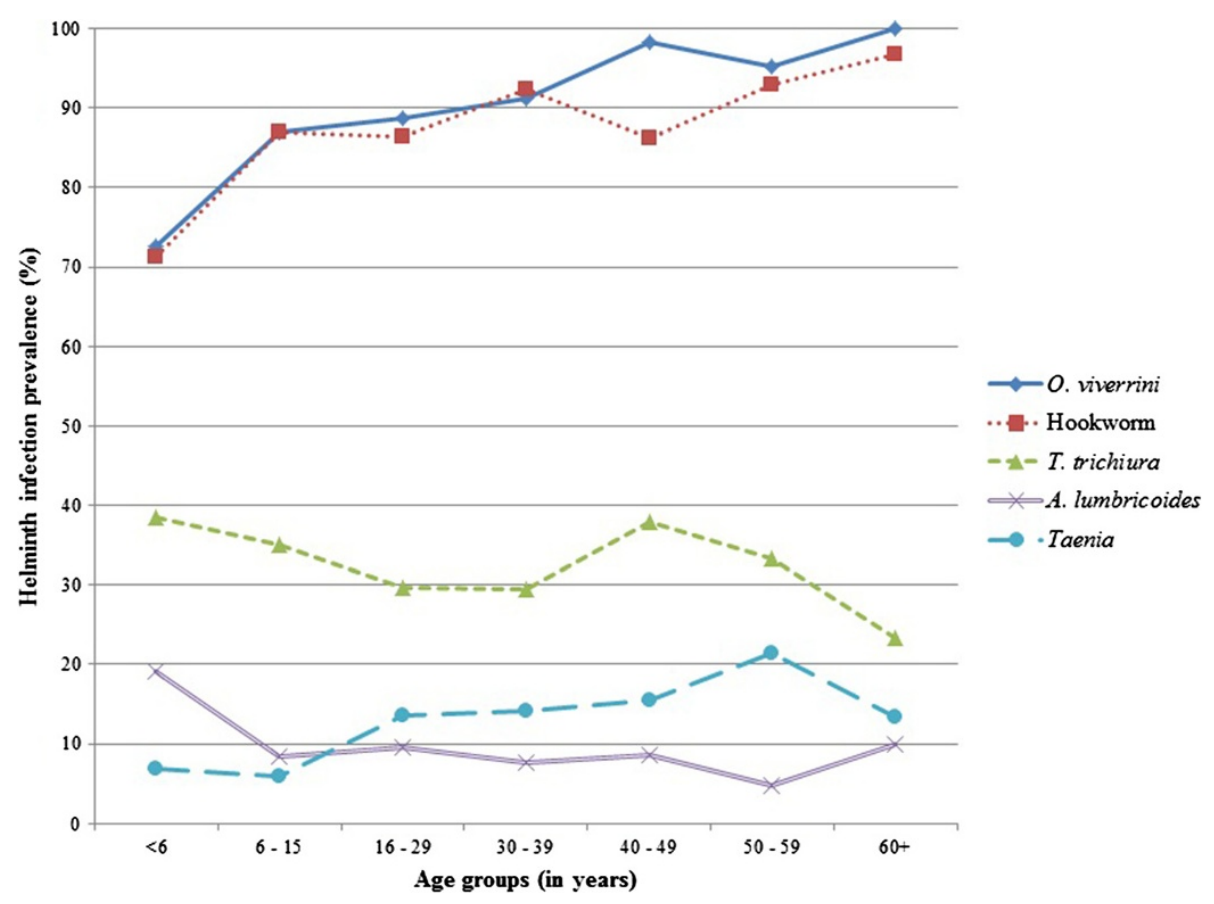

Figure 3 Helminth infection rate by age groups, Saravane district, $2010(n=574)$.

to eat raw small cyprinoid fish salad, while two other men stated that eating raw fish dishes was their habit. One woman (aged 56 years) in Nongbouayai village said: "We have the habit of eating raw fish dishes such as Koi Pa, Lap Pa Nuew, Koi Phan, Koi Cham because, on the one hand, we would like to change the style of cooking our usual dishes but, on the other hand, this type of dish like Lap Pa Nuew can be easily prepared and served for many people and is sufficient for all members in the family, even if we only could catch a small amount of fish". Eight women confirmed that women mostly prepared raw fish dishes. Some respondents (16.4\%) reported consuming Som $\mathrm{Pa}$ (raw sour rice-fermented fish). Eight participants stated that this dish is rarely consumed.

The frequency of eating raw fish dishes depends on the availability of fish in the village. Three-quarters of participants $(75.8 \%)$ reported that during the rainy season and

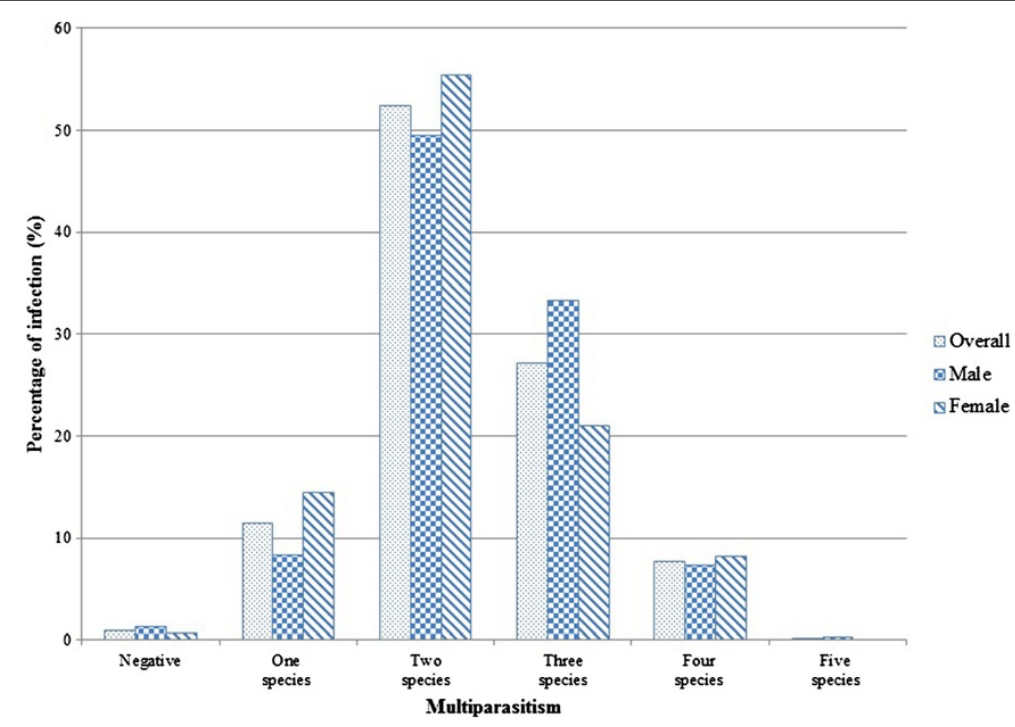

Figure 4 Multiple helminth infections in study population by gender in Saravane district, $2010(n=574)$. 
Table 2 Summary results for logistic regression analysis for the association between risk factors and helminth infections $(\mathbf{n}=\mathbf{5 7 4})$

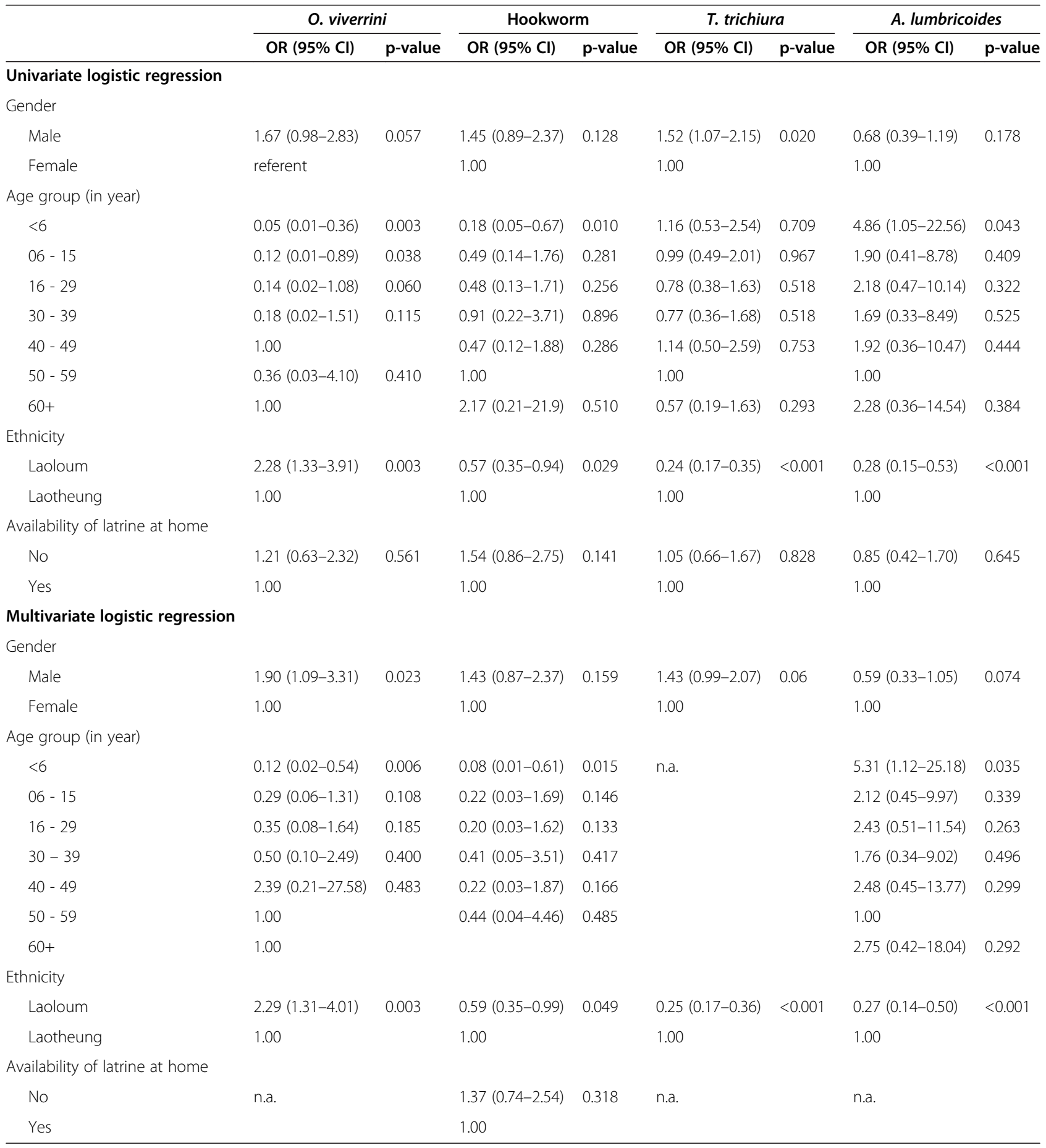

thereafter, people more frequently eat raw fish dishes due to an abundance of fish in the ponds, rivers and rain-fed paddy fields. During the dry season, most people do not eat it more than once per month. A man from Chantai village (aged 45 years) stated that, "...if fish were always available throughout the year, we would eat Koi Pa, Lap Pa Nuew, Koi Phan and Koi Cham every day".

When asked about personal hygiene, 46.3\% mentioned that hand washing before eating was common. However, one woman (aged 41 years) mentioned that she sometimes forgot to wash her hands, particularly when very 
Table 3 Knowledge of helminthiases among heads of households in 10 villages in Saravane district, $2010(n=130$ )

\begin{tabular}{|c|c|c|c|c|}
\hline Item & Total & Male $(n=69)$ & Female $(n=61)$ & p-value \\
\hline \multicolumn{5}{|l|}{ Knowledge about liver fluke } \\
\hline Heard about liver fluke & $49(37.7)$ & 46.4 & 27.9 & 0.030 \\
\hline Transmission route for liver fluke infection: & & $\mathrm{n}=32$ & $n=17$ & \\
\hline Eating raw fish dishes (Koi Pa, Lap Pa Nuew) & $29(59.2)$ & 65.6 & 47.1 & 0.208 \\
\hline Eating raw sour fermented fish & $12(24.5)$ & 18.7 & 35.3 & 0.200 \\
\hline Eating raw fermented fish & $12(24.5)$ & 28.1 & 17.6 & 0.503 \\
\hline Eating pickled fish & $6(12.2)$ & 12.5 & 11.8 & 1.000 \\
\hline Prevention of liver fluke infection: & & & & 0.374 \\
\hline Not eating any raw food: meat, shrimp etc. & $21(42.9)$ & 40.6 & 47.1 & \\
\hline Avoiding raw fish consumption & $7(14.3)$ & 18.7 & 5.9 & \\
\hline Maintaining good personal hygiene and use latrine & $2(4.1)$ & 6.3 & 0 & \\
\hline Taking deworming medicine & $2(4.1)$ & 3.1 & 5.9 & \\
\hline Avoiding cigarettes and alcohol & $1(2.0)$ & 3.1 & 0 & \\
\hline Seeking health care & $1(2.0)$ & 0 & 5.9 & \\
\hline \multicolumn{5}{|l|}{ Knowledge about soil-transmitted helminths } \\
\hline Heard about hookworm & $11(8.5)$ & 5.8 & 11.5 & 0.346 \\
\hline Transmission route for hookworm infection: & & $\mathrm{n}=4$ & $n=7$ & 0.448 \\
\hline Eating raw meat, fish, vegetables & $2(18.2)$ & 25.0 & 14.3 & \\
\hline Infection through food & $1(9.1)$ & 25.0 & 0 & \\
\hline No idea & $8(72.7)$ & 50.0 & 85.7 & \\
\hline Prevention of hookworm infection: & & & & 0.288 \\
\hline Taking deworming drugs & $1(9.1)$ & 0 & 14.3 & \\
\hline Avoiding raw foodstuff consumption & $3(27.3)$ & 50 & 14.3 & \\
\hline Maintaining good personal hygiene & $2(18.2)$ & 25 & 0 & \\
\hline Heard about whipworm & $2(1.5)$ & 1.4 & 1.6 & 1.000 \\
\hline Transmission route for whipworm infection & 0 & 0 & 0 & \\
\hline Prevention of whipworm infection & 0 & 0 & 0 & \\
\hline Heard about roundworm & $92(70.8)$ & 69.6 & 72.1 & 0.748 \\
\hline Knowledge about transmission route: & & $\mathrm{n}=48$ & $n=44$ & \\
\hline Hand washing before eating & $8(8.7)$ & 10.4 & 6.8 & 0.716 \\
\hline Eating raw food & $84(91.3)$ & 89.6 & 93.2 & 0.716 \\
\hline \multicolumn{5}{|l|}{ Prevention of roundworm infection: } \\
\hline Hand washing before eating & 19 (20.6) & 27.1 & 13.6 & 0.111 \\
\hline Hand washing after using latrine & $8(8.7)$ & 10.4 & 6.8 & 0.716 \\
\hline Taking deworming drug & $11(11.9)$ & 8.3 & 15.9 & 0.341 \\
\hline Eating cooked food & $33(45.2)$ & 58.9 & 41.2 & 0.129 \\
\hline
\end{tabular}

hungry after an intense period of working in rice fields. Ten participants said that no one in the village washes his/her hands after defecation.

When discussing the benefits of hand washing before eating, $29.9 \%$ of participants thought that it would not prevent any worm infections or any other diseases; $14.9 \%$ did it just to keep the hands clean; only a few participants (4.5\%) linked it to health benefits.
Open defecation is very common and was mentioned by 16 of the 67 FGD participants (23.9\%). When asked about latrine use, one man (aged 55 years) in Chantai village said that he had asked the government to support latrine construction because the surrounding forest is going to be "finished" soon, and they will not have a place to defecate anymore. Three men and one woman disliked the use of pit latrines due to their bad smell. Two men said: "I feel uncomfortable if I use the toilet 
Table 4 Risk behaviour of heads of households in Saravane district, $2010(n=130)$

\begin{tabular}{|c|c|c|c|c|}
\hline Risky behaviour & Total & Male $(n=69)$ & Female $(n=61)$ & p-value \\
\hline \multicolumn{5}{|l|}{ Habit of eating raw food } \\
\hline Eat raw fish dishes (Koi Pa, Lap Pa Nuew) & $98(75.4)$ & 91.3 & 57.4 & $<0.001$ \\
\hline Eat sour fermented fish (Som $\mathrm{Pa}$, Som $\mathrm{Pa}$ Noy/Som $\mathrm{Pa}$ Chom) & $59(45.4)$ & 56.5 & 32.8 & 0.007 \\
\hline Eat raw fermented fish (Pa Daek, Pa Daek Nam) & $82(68.1)$ & 75.4 & 49.2 & 0.002 \\
\hline Eat raw sausages of pork (Som Mou Dip) & $43(33.1)$ & 46.4 & 18.0 & 0.001 \\
\hline Eat raw beef salad (Lap Xin Dip) & $93(71.5)$ & 88.4 & 52.5 & $<0.001$ \\
\hline Eat raw vegetables & $124(95.4)$ & 98.5 & 91.8 & 0.098 \\
\hline Cleaning vegetables before eating & $123(94.6)$ & 98.5 & 90.2 & 0.051 \\
\hline \multicolumn{5}{|l|}{ Personal hygiene } \\
\hline Hand washing before eating & $127(97.7)$ & 97.1 & 98.4 & 0.663 \\
\hline Hand washing after defecation & $108(83.1)$ & 81.2 & 85.2 & 0.535 \\
\hline Hand washing with soap & $15(11.5)$ & 5.8 & 18.0 & 0.029 \\
\hline Wearing shoes when get outside/going to bush & $119(91.5)$ & 89.9 & 93.4 & 0.540 \\
\hline \multicolumn{5}{|l|}{ Sanitation facility } \\
\hline Having latrine at home & $21(16.1)$ & 11.6 & 21.3 & 0.133 \\
\hline Using latrine every time & $16(76.2)$ & 50.0 & 92.3 & 0.047 \\
\hline Using latrine sometime & $4(19.0)$ & 37.5 & 7.7 & \\
\hline Not using latrine at all & $1(4.8)$ & 12.5 & 0 & \\
\hline
\end{tabular}

because I cannot get used to it. I am afraid all the time that others will open the toilet's door". Another one said that, "...what do we do if the toilet is full? I would say that to defecate in the forest is therefore a more convenient way".

During the discussion about the general benefits of latrines, only $14.9 \%$ participants thought that the use of latrines could prevent diseases from being transmitted in their village; four FGD participants thought that animals in the village would not eat human faeces any more if they had latrines. For instance, one woman aged 58 years from Naphengyai village said that, "...animals would not eat human faeces and chickens would not die anymore..."; the remaining seven participants mentioned that the villages would be cleaner and latrine use more convenient.

\section{Direct observation results}

In two of the ten study villages, we could directly observe fish dish preparation during our field work. In Dongkoneua village, the village committee obtained the fish from the nearby village market and prepared a welcome meal for our team while we were working in their village. The food served during that meal was well cooked. In Chantai village, household members caught fish from a nearby river and prepared raw sticky fish salad (Lap Pa Nuew). The team was told that Lap $\mathrm{Pa}$ Nuew must be served with raw fish.

Of ten study villages, five had at least a few latrines. However, in these five villages, fewer than $20 \%$ of households owned a latrine. Only one of four FGD villages (Naphengyai) had latrines. We looked at eleven latrines. Three had never been used before, while three others could only be used at night (i.e. they had no walls or roof and one was far from the source of water). Five latrines were used regularly (three were owned by village heads and two were owned by village health volunteers). We observed pigs, cows and buffalos straying freely in the study villages. Walking barefoot outside of the houses was very common, particularly for children.

\section{Discussion}

This study deepened our understanding of helminth infections and multiparasitism in relation to knowledge, attitudes and practices in Southern Laos, where helminth infections are highly endemic. We conducted a cross-sectional survey in ten randomly selected villages in Saravane district. Stool examinations were performed on all members (aged $\geq 2$ years) of selected households. At the same time, in-depth interviews with the heads of households, FGDs and direct observations were performed to better understand knowledge, attitudes and practices related to these infections.

Our findings show that trematode infections, i.e. O. viverrini infections, were highly prevalent (88.7\%). In fact, prevalence rates were one and a half time higher than indicated in a previous assessment in 2004 (58.5\%) [23], which might be explained by the fact that no community control activities were conducted between the two assessments. The current study employed a rigorous 
Table 5 Summary of focus group discussions, Saravane district, $2010(n=67)$

\begin{tabular}{|c|c|c|c|}
\hline Items & n (\%) & Male $(n=32)$ & Female $(n=35)$ \\
\hline \multicolumn{4}{|l|}{ Knowledge about worm infection: } \\
\hline Heard about liver fluke "san tap" or "pha yat bai mai nai tap" & $34(50.7)$ & 34.4 & 65.7 \\
\hline \multicolumn{4}{|l|}{ How to get infection with $O$. viverrini: } \\
\hline Eating raw vegetables & $3(4.5)$ & 3.1 & 5.7 \\
\hline Not maintaining good personal hygiene: hand washing, nail clipping, unclean environment & $8(11.9)$ & 15.6 & 11.4 \\
\hline Eating any raw food: meat, snail, shrimp & $29(43.3)$ & 34.4 & 51.4 \\
\hline Eating raw fish dishes (Koi Pa, Lap Pa Nuew) & $8(11.9)$ & 12.5 & 11.4 \\
\hline Heard about roundworm & $52(77.6)$ & 84.4 & 71.4 \\
\hline \multicolumn{4}{|l|}{ How to get infection with roundworm: } \\
\hline It just occurs in our body & $2(2.9)$ & 0 & 5.7 \\
\hline Eat any raw: meat, fish, shrimp, vegetables & $7(10.4)$ & 15.6 & 5.7 \\
\hline $\begin{array}{l}\text { Not maintaining good personal hygiene: not hand washing before eating, } \\
\text { not clipping nails, flies touching our food, drinking dirty water }\end{array}$ & $16(23.9)$ & 18.7 & 28.6 \\
\hline Heard about hookworm & $3(4.5)$ & 6.2 & 2.8 \\
\hline Do not know & $3(4.5)$ & 6.2 & 2.8 \\
\hline Heard about whipworm & $2(2.9)$ & 6.2 & 0 \\
\hline Do not know & $2(2.9)$ & 6.2 & 0 \\
\hline Heard about tapeworm & $33(49.3)$ & 46.9 & 51.4 \\
\hline Eating raw food & $8(11.9)$ & 21.9 & 2.8 \\
\hline Eating raw fish dishes & $33(49.3)$ & 53.1 & 45.7 \\
\hline \multicolumn{4}{|l|}{ Perceptions of latrine use: } \\
\hline Village will not be dirty & $15(22.4)$ & 34.4 & 11.4 \\
\hline Flies do not touch our food anymore & $4(5.9)$ & 12.5 & 0 \\
\hline Prevention of diseases/no transmission of diseases & $10(14.9)$ & 18.8 & 11.4 \\
\hline Animals will not eat human faeces & $6(8.9)$ & 0 & 17.1 \\
\hline Convenience (not getting wet from rain, convenience during the night and when sick) & $13(19.4)$ & 6.2 & 31.4 \\
\hline Perceived liver fluke as health problem in community & $6(8.9)$ & 12.5 & 5.7 \\
\hline \multicolumn{4}{|l|}{ Practices personal hygiene: } \\
\hline Hand washing before eating & $31(46.3)$ & 31.2 & 60.0 \\
\hline Saying that most of people in village do not wash hands with soap & $10(14.9)$ & 0 & 28.6 \\
\hline \multicolumn{4}{|l|}{ Perceptions of personal hygiene: } \\
\hline Hands will be clean if we wash hands before eating & $10(14.9)$ & 0 & 28.6 \\
\hline We will be healthy if we wash hands before eating & $3(4.5)$ & 9.4 & 0 \\
\hline We can not get any worm infections or diseases if we wash our hands before eating & $20(29.9)$ & 28.1 & 31.4 \\
\hline \multicolumn{4}{|l|}{ Deworming in community: } \\
\hline Getting dewormed & $37(55.2)$ & 75.0 & 37.1 \\
\hline Treatment of tapeworm with traditional medicine & $19(28.3)$ & 43.7 & 14.3 \\
\hline Treatment with modern medicine & $18(26.9)$ & 31.2 & 22.8 \\
\hline Treatment of roundworm using modern medicine & $2(2.9)$ & 2.9 & 0 \\
\hline
\end{tabular}

diagnostic procedure using two stool samples per person, which certainly contributes to explaining the higher prevalence rate of the current assessment. In addition, most individuals had multiple infections with two helminth species and a quarter was infected with more than three species. Co-infections were most frequent with hookworm, which was the second most frequent helminth infection found in our study (77.3\%).

Our interview results reveal that household heads have very limited knowledge of $O$. viverrini infection, its route 
of transmission and potential means of prevention. The habit of eating raw and insufficiently cooked fish was a very common practice and open defecation was widespread. Nevertheless, some household heads recognised the benefits of latrine use and gave reasons for not using them. During the discussions, participants mentioned that they disliked using latrines because they were either not used to it or did not know what to do when latrines were filled up. Even among people who had a latrine, not all of them used latrines regularly. Interestingly, women used latrines significantly more than men, indicating that they see a much greater benefit from having latrines in a household. Direct observations fully supported this finding. Hence, future sanitation promotion community interventions should particularly focus on women in target communities.

Our triangulated methodological approach (i.e. stool examination, interview with heads of households, FGDs with community members and direct observation) allowed us to confirm findings from different sources of data and, hence, show the strong relationship between the high prevalence rate of helminth infections and the corresponding low level of awareness and knowledge of selected community members. However, our helminth infection assessment was performed with a Kato-Katz technique, which does not allow one to distinguish between $O$. viverrini and minute intestinal flukes (MIF) [26]. The most recent research based on purging infected individuals and molecular diagnostic tools showed that in Southern Laos, a considerable amount of MIF is present $[27,28]$. Thus, it is likely that the O. viverrini infection rate is over-reported in the current study. However, the route of transmission and preventive measures for these parasites are the same. Therefore, our findings of low community knowledge and awareness of worm infections is still valid.

Our research shows a distinct link between the prevalence of hookworm infection and the household heads' knowledge of hookworm infections. A very high hookworm infection rate $(86.6 \%)$ was observed and increased with age, from $71.2 \%$ in the youngest age group to $96.7 \%$ in the oldest age group. Conversely, household heads showed an extremely low level of knowledge of hookworm, e.g. awareness of infection risk through direct contact with soil. The majority of household heads reported wearing shoes outside but investigators directly observed that walking barefoot outside was very common in all villages. In addition, animal excreta originating from free straying animals such as pigs, cows and buffalos were observed in all surveyed villages. A recent study conducted in rural areas of central Thailand confirmed that walking barefoot outside and keeping livestock such as buffalos around the house were risk factors for hookworm infection [29]. Hookworm infection is known to be an important cause of iron deficiency, affecting the physical and cognitive development of children [9]. Therefore, building awareness of the modes of transmission, the relationship between infection and disease, and preventative measures needs to be addressed in these affected communities.

The Lao government has made considerable efforts to control STHs in women at reproductive age, in children under five years of age and in primary school children by providing regular mass treatment with mebendazole twice a year (single oral dose $500 \mathrm{mg}$ ) [30]. Against this backdrop, it is most astonishing that hookworm infection prevalence remains high. A high re-infection rate is a plausible explanation. Adequate health education messages and alternative communication channels, for instance peer education of mothers and primary school children, could address these issues. Another likely reason for the persistently high hookworm infection rate is the use of mebendazole instead of albendazole. Mebendazole has shown very low curative effects in recent studies in Southeast Asia [31,32] and therefore, albendazole (single oral dose of $400 \mathrm{mg}$ ) should be used in deworming campaigns.

O. viverrini infection is acquired through consuming raw fish $[23,33,34]$. In our study, we found that household heads have poor knowledge of liver fluke infection, including its transmission mode and means of prevention. This low level of awareness is certainly one reason why raw fish eating practices in these communities are still prevalent and have not changed substantially since the last assessment made by Sayasone and colleagues in 2004 [23]. A study conducted in northeast Thailand, where $O$. viverrini infection rate is very high, identified viable metacercariae in raw fish dishes such as Koi $\mathrm{Pa}$ and Som $\mathrm{Pa}$ [35]. Therefore, a prerequisite of preventing $O$. viverrini infection in an efficient way is to decrease raw fish consumption. Awareness building is the first step in this process, using adequate health education messages and approaches.

The populous, rural communities of the Laoloum people show a very rich cultural life, with high and regular intensity of religious ceremonies and social occasions (e.g. life stage and agricultural events), where raw fish dishes are a prominent marker of commensality and underline the importance of ethnic and religious belonging [36]. In contrast, Laotheung communities - and many of them adhere to local religious beliefs - do not, in general, engage in the multitude of elaborate ceremonial practices, which include the mandatory consumption of raw or insufficiently cooked fish. Furthermore, the Laoloum people and their villages have a much better, and thus more frequent, access to raw fish and raw fish dishes, particularly at markets, food stalls, in shops and through street vendors, than do the Laotheung who tend to live in 
somewhat remote areas without well-developed road networks and easy access to urban centres. Direct observation revealed that environmental conditions also contribute to the increased $O$. viverrini infection rate among the riparian Laoloum people: they live mainly along big rivers and streams with a large fish stock; the abundance of freshwater fish leads to regular consumption of raw fish [36]. In contrast, Laotheung communities live in a mostly hilly and rugged physical environment and do not have this rich quantity and quality of fish in the creeks; consequently, they consume raw fish less frequently.

This study indicates that men have a higher risk of being infected with $O$. viverrini than women do. This quantitative result is consistent with the findings from FGDs where men discussed their preference for eating raw fish dishes such as Koi $\mathrm{Pa}$, Koi Pa Siew, and Lap Pa Nuew. Eating raw fish dishes is their habit and they would eat this food every day if fish were always available. Furthermore, men, as social, economic, political and religious representatives of their households and kin, participate in many more public and official events in the community than do women, where raw or insufficiently cooked fish dishes are usually served and consumed in commensality. The consumption of raw fish dishes is part of one's social obligations and integration in Laos [36]. A study by Strandgaard and colleagues in Vientiane province obtained similar findings where more men ate raw fish dishes than women did [37]. It is also known that in endemic liver fluke areas a slightly higher prevalence rate of $O$. viverrini infection was observed in men [38]. Moreover, we found that more men harboured different worm species than women did. However, previous assessment in this district by Sayasone and colleagues in 2004 did not show a difference of infection rates between genders [23]. Appropriate health messages regarding flukes and STHs need to address these gender differences and tackle men's riskier consumption pattern.

Our research confirms previous results that $O$. viverrini infection prevalence increases with age [23,25,39]. Although our study found that children $<6$ years had a low risk of $O$. viverrini infection compared to other age groups, these children showed a much higher $O$. viverrini infection rate than found in a previous study in 2000 (72.6\% versus 27.9\%) [39]. In addition, the majority of $O$. viverrini-infected children aged 16 and under was co-infected with hookworm (74.7\%). Regarding helminth infection, our study found that $49.2 \%$ of heads of households allowed their children to share any raw fish dish with adults once they could eat by themselves, particularly at age three (mentioned by $67.7 \%$ ) and at age two (6.1\%). This long-term exposure (from childhood) to O. viverrini infection poses serious health problems: O. viverrini is carcinogenic for cholangiocarcinoma [8], particularly when the infected person turns 35 years or older $[32,40]$. The interrelation of $O$. viverrini infection and age becomes a serious public health issue and requires an intergenerational (i.e. acting between children and their parents) and transgenerational approach (i.e. acting across multiple generations such as children and elderly people) to health education, for instance, targeting health messages and measures to each different age group.

Although Saravane province, like other provinces in Laos, benefits from rapid economic and infrastructural development, helminth infections in general and fluke infections in particular remain an important public health concern. To address it appropriately, a communitywide intervention must be initiated to ensure access to treatment and health education to increase knowledge of worm infections. Based on our findings, a combination of informal and formalised health education activities might be best suited to broaden local people's awareness and to promote adoption of healthy practices related to helminth infections.

\section{Conclusions}

This study discerned the relationship between helminth infection rates and quantity and the level of awareness of parasitic infections and its risk factors in endemic liver fluke areas in Saravane district. It highlights that helminth infection, particularly fluke and hookworm infections, imply a high burden, followed by $T$. trichiura, Taenia sp. and A. lumbricoides, notably in communities where multiple helminth infections exist. However, specific knowledge and awareness of helminth infections was very limited, particularly regarding the mode of transmission and means of prevention. Consumption of raw and insufficiently cooked fish was widely practiced because these fish dishes are deeply rooted in the local culture of food and nutrition, particularly among the Laoloum. We observed poor personal hygiene practices and unreliable village sanitation; only a few households have access to a latrine but not everybody who has a latrine uses it regularly. This study calls for local authorities and communities in Saravane district to integrate actions to address helminth infections by building awareness and strengthening knowledge about worm infections and practices related to these infections. Furthermore, this research adds to the much-needed arsenal of mixed quantitative and qualitative methodological approaches in helminth studies. Qualitative methods in combination with quantitative baseline studies of infection rates shed light on social determinants of helminth infection as well as on cultural processes and community and individual health practices in regard to helminth vulnerability and reveal significant relations between prevalence rates, reasons for infection and appropriate problem solving measures. 


\section{Competing interests}

The authors declare that they have no competing interests and that the sponsors had no role in the study design, data collection and analysis, decision to publish, or preparation of the manuscript.

\section{Authors' contributions}

KP and PO conceived the study idea, and designed and analysed the data, and interpreted results together with PVE. KP coordinated and conducted field work and drafted the manuscript; VX carried out data collection and sample collection; Y carried out laboratory work; KA had overall responsibility for data collection; PVE and PO revised the manuscript. All authors read and approved the final submission.

\section{Acknowledgements}

We would like to express our sincere thanks to the authorities, in particular the Ministry of Health, the National Centre of Malariology, Parasitology and Entomology, the Saravane Provincial Health Department, the Saravane District Health Office, the local authorities in the study villages, the participants involved in this study and our research team. Special thanks go to Phonepasong Ayé Soukhathammavong, Armelle Forrer, Audrey Bochaton, and Amena Briet for their help. We are grateful for generous financial support from the World Health Organisation (Geneva), and Rudolf Geigy Foundation and Stiftung für freiheitliche Medizin (Basel, Switzerland).

\section{Author details}

${ }^{1}$ National Institute of Public Health, Ministry of Health, Vientiane, Lao PDR. ${ }^{2}$ Department of Epidemiology and Public Health, Swiss Tropical and Public Health Institute, P.O. Box, CH-4002, Basel, Switzerland. '3niversity of Basel, Basel, Switzerland. ${ }^{4}$ Institute of Social Anthropology, Basel, Switzerland. ${ }^{5}$ University of Health Science, Ministry of Health, Vientiane, Lao PDR.

Received: 25 September 2013 Accepted: 10 November 2013 Published: 14 November 2013

\section{References}

1. Utzinger J, Bergquist R, Olveda R, Zhou XN: Important helminth infections in Southeast Asia diversity, potential for control and prospects for elimination. Adv Parasitol 2010, 72:1-30.

2. Fürst T, Keiser J, Utzinger J: Global burden of human food-borne trematodiasis: a systematic review and meta-analysis. Lancet Infect Dis 2012, 12:210-221.

3. Sayasone S, Vonghajack Y, Vanmany M, Rasphone O, Tesana S, Utzinger J, Akkhavong K, Odermatt P: Diversity of human intestinal helminthiasis in Lao PDR. Trans R Soc Trop Med Hyg 2009, 103:247-254.

4. Sayasone S, Mak TK, Vanmany M, Rasphone O, Vounatsou P, Utzinger J, Akkhavong K, Odermatt P: Helminth and intestinal protozoa infections, multiparasitism and risk factors in Champasack province, Lao People's Democratic Republic. PLoS Negl Trop Dis 2011, 5:e1037.

5. Keiser J, Utzinger J: Food-borne trematodiasis: current chemotherapy and advances with artemisinins and synthetic trioxolanes. Trends Parasitol 2007, 23:555-562

6. Keiser J, Utzinger J: Efficacy of current drugs against soil-transmitted helminth infections: systematic review and meta-analysis. JAMA 2008, 299:1937-1948.

7. Sithithaworn P, Andrews RH, Nguyen VD, Wongsaroj T, Sinuon M, Odermatt P, Nawa Y, Liang S, Brindley PJ, Sripa B: The current status of opisthorchiasis and clonorchiasis in the Mekong Basin. Parasitol Int 2012, 61:10-16.

8. Bouvard V, Baan R, Straif K, Grosse Y, Secretan B, El GF, brahim-Tallaa L, Guha N, Freeman C, Galichet L, et al: A review of human carcinogens-Part B: biological agents. Lancet Oncol 2009, 10:321-322.

9. Hotez PJ, Bundy DAP, Beegle K, Brooker S, Drake L, de Silva N, Montresor A, Engels $D$, Jukes M, Chitsulo L, et al: Helminth infections: soil-transmitted helminth infections and schistosomiasis. In Disease Control Priorities in Developing Countries. 2nd edition. Edited by Jamison DT. Washington (DC): World Bank; 2006. Chapter 24.

10. WHO Expert committee: Prevention and control of schistosomiasis and soil-transmitted helminthiasis. World Health Organ Tech Rep Ser 2002, 912:i-vi. 1-57.

11. WHO/FAO: Joined workshop on food-borne trematode infections in Asia. Hanoi, Vietnam: WHO Report; 2004.
12. Lovis L, Mak TK, Phongluxa K, Aye SP, Vonghachack Y, Keiser J, Vounatsou P, Tanner M, Hatz C, Utzinger J, et al: Efficacy of praziquantel against Schistosoma mekongi and Opisthorchis viverrini: a randomized, single-blinded dose-comparison trial. PLOS Negl Trop Dis 2012, 6:e1726.

13. Phan VT, Ersboll AK, Do DT, Dalsgaard A: Raw-fish-eating behavior and fishborne zoonotic trematode infection in people of northern Vietnam. Foodborne Pathog Dis 2011, 8:255-260.

14. Cantey PT, Rout J, Rao G, Williamson J, Fox LM: Increasing compliance with mass drug administration programs for lymphatic filariasis in India through education and lymphedema management programs. PLOS Negl Trop Dis 2010, 4:e728.

15. Gunawardena S, Ismail M, Bradley M, Karunaweera N: Factors influencing drug compliance in the mass drug administration programme against filariasis in the Western province of Sri Lanka. Trans $R$ Soc Trop Med Hyg 2007, 101:445-453.

16. Nuwaha F, Okware J, Ndyomugyenyi R: Predictors of compliance with community-directed ivermectin treatment in Uganda: quantitative results. Trop Med Int Health 2005, 10:659-667.

17. Yirga D, Deribe K, Woldemichael K, Wondafrash M, Kassahun W: Factors associated with compliance with community directed treatment with ivermectin for onchocerciasis control in Southwestern Ethiopia. Parasit Vectors 2010, 3:48

18. Forrer A, Sayasone S, Vounatsou P, Vonghachack Y, Bouakhasith D, Vogt S, Glaser R, Utzinger J, Akkhavong K, Odermatt P: Spatial distribution of, and risk factors for, Opisthorchis viverrini infection in southern Lao PDR. PLoS Negl Trop Dis 2012, 6:e1481.

19. Sayasone S, Tesana S, Utzinger J, Hatz C, Akkhavong K, Odermatt P: Rare human infection with the trematode Echinochasmus japonicus in Lao PDR. Parasitol Int 2009, 58:106-109.

20. Soukhathammavong $P$, Odermatt $P$, Sayasone $S$, Vonghachack $Y$, Vounatsou $P$, Hatz C, Akkhavong K, Keiser J: Efficacy and safety of mefloquine, artesunate, mefloquine-artesunate, tribendimidine, and praziquantel in patients with Opisthorchis viverrini: a randomised, exploratory, openlabel, phase 2 trial. Lancet Infect Dis 2011, 11:110-118.

21. Lao MOH: Diagnosis and Treatment in district hospitals. Lao PDR: Institut de la Francophonie pour la médicine tropicale, Ministry of Health; 2004.

22. Rim HJ, Chai JY, Min DY, Cho SY, Eom KS, Hong SI, Sohn WM, Yong TS, Deodato G, Hanne S, et al: Prevalence of intestinal parasite infections on a national scale among primary schoolchildren in Laos. Parasitol Res 2003, 91:267-272

23. Sayasone $S$, Odermatt $P$, Phoumind $N$, Vongsaravane $X$, Sensombath $V$, Phetsouvanh R, Choulamany X, Strobel M: Epidemiology of Opisthorchis viverrini in a rural district of southern Lao PDR. Trans $R$ Soc Trop Med Hyg 2007, 101:40-47.

24. Katz N, Chaves A, Pellegrino J: A simple device for quantitative stool thicksmear technique in Schistosomiasis mansoni. Rev Inst Med Trop Sao Paulo 1972, 14:397-400.

25. Maleewong W, Intapan P, Wongwajana S, Sitthithaworn P, Pipitgool V, Wongkham C, Daenseegaew W: Prevalence and intensity of Opisthorchis viverrini in rural community near the Mekong River on the Thai-Laos border in northeast Thailand. J Med Assoc Thai 1992, 75:231-235.

26. Lovis L, Mak TK, Phongluxa K, Soukhathammavong P, Sayasone S, Akkhavong K, Odermatt P, Keiser J, Felger I: PCR Diagnosis of Opisthorchis viverrini and Haplorchis taichui Infections in a Lao Community in an area of endemicity and comparison of diagnostic methods for parasitological field surveys. J Clin Microbiol 2009, 47:1517-1523.

27. Chai JY, Yong TS, Eom KS, Min DY, Jeon HK, Kim TY, Jung BK, Sisabath L, Insisiengmay B, Phommasack B, et al: Hyperendemicity of Haplorchis taichui Infection among Riparian People in Saravane and Champasak Province, Lao PDR. Korean J Parasitol 2013, 51:305-311.

28. Rim HJ, Sohn WM, Yong TS, Eom KS, Chai JY, Min DY, Lee SH, Hoang EH, Phommasack $B$, Insisiengmay S: Fishborne trematode metacercariae in Luang Prabang, Khammouane, and Saravane Province, Lao PDR. Korean J Parasitol 2013, 51:107-114.

29. Jiraanankul V, Aphijirawat W, Mungthin M, Khositnithikul R, Rangsin R, Traub RJ, Piyaraj P, Naaglor T, Taamasri P, Leelayoova S: Incidence and risk factors of hookworm infection in a rural community of central Thailand. Am J Trop Med Hyg 2011, 84:594-598.

30. Phommasack B, Saklokham K, Chanthavisouk C, Nakhonesid-Fish V, Strandgaard H, Montresor A, Shuey DA, Ehrenberg J: Coverage and costs 
of a school deworming programme in 2007 targeting all primary schools in Lao PDR. Trans R Soc Trop Med Hyg 2008, 102:1201-1206.

31. Soukhathammavong PA, Sayasone S, Phongluxa K, Xayaseng V, Utzinger J, Vounatsou P, Hatz C, Akkhavong K, Keiser J, Odermatt P: Low efficacy of single-dose albendazole and mebendazole against hookworm and effect on concomitant helminth infection in Lao PDR. PLoS Negl Trop Dis 2012, 6:e1417.

32. Steinmann P, Utzinger J, Du ZW, Jiang JY, Chen JX, Hattendorf J, Zhou $H_{\text {, }}$ Zhou XN: Efficacy of single-dose and triple-dose albendazole and mebendazole against soil-transmitted helminths and Taenia spp.: a randomized controlled trial. PLoS One 2011, 6:e25003.

33. Grundy-Warr C, Andrews RH, Sithithaworn P, Petney TN, Sripa B, Laithavewat L, Ziegler AD: Raw attitudes, wetland cultures, life-cycles: socio-cultural dynamics relating to Opisthorchis viverrini in the Mekong Basin. Parasitol Int 2012, 61:65-70.

34. Tomokawa S, Kobayashi T, Pongvongsa T, Nisaygnang B, Kaneda E, Honda S, Moji K, Boupha B: Risk factors for Opisthorchis viverrini infection among schoolchildren in Lao PDR. Southeast Asian J Trop Med Public Health 2012, 43:574-585.

35. Prasongwatana J, Laummaunwai P, Boonmars T, Pinlaor S: Viable metacercariae of Opisthorchis viverrini in northeastern Thai cyprinid fish dishes-as part of a rational program for control of $O$. viverrini-associated cholangiocarcinoma. Parasitol Res 2013, 112:1323-1327.

36. Xayaseng V, Phongluxa $K$, van Eeuwijk $P$, Akkhavong $K$, Odermatt P: Raw fish consumption in liver fluke endemic areas in rural southern Laos. Acta Trop 2013, 127:105-111.

37. Strandgaard H, Johansen MV, Agaard-Hansen J, Petlueng P, Ornbjerg N: Local perceptions and practices in regard to opisthorchiasis in two villages in Lao PDR. Southeast Asian J Trop Med Public Health 2008, 39:19-26.

38. Sithithaworn P, Haswell-Elkins M: Epidemiology of Opisthorchis viverrini. Acta Trop 2003, 88:187-194.

39. Kobayashi J, Vannachone B, Sato Y, Manivong K, Nambanya S, Inthakone S: An epidemiological study on Opisthorchis viverrini infection in Lao villages. Southeast Asian J Trop Med Public Health 2000, 31:128-132.

40. Sriamporn S, Pisani P, Pipitgool V, Suwanrungruang K, Kamsaard S, Parkin DM: Prevalence of Opisthorchis viverrini infection and incidence of cholangiocarcinoma in Khon Kaen, Northeast Thailand2. Trop Med Int Health 2004, 9:588-594.

doi:10.1186/1756-3305-6-328

Cite this article as: Phongluxa et al:: Helminth infection in southern

Laos: high prevalence and low awareness. Parasites \& Vectors 2013 6:328.

\section{Submit your next manuscript to BioMed Central and take full advantage of:}

- Convenient online submission

- Thorough peer review

- No space constraints or color figure charges

- Immediate publication on acceptance

- Inclusion in PubMed, CAS, Scopus and Google Scholar

- Research which is freely available for redistribution 